\title{
EMIGRAZIONE ARTIGIANA STAGIONALE DALLE MONTAGNE DEL BELLUNESE AI PAESI DELL'EUROPA CENTRO-ORIENTALE TRA FINE OTTOCENTO E INIZI NOVECENTO
}

\author{
Laura Campanale \\ ITT Mazzotti - Treviso \\ dottlaura@yahoo.it
}

\section{Riassunto}

Il presente contributo tratta dei flussi migratori stagionali che tra fine Ottocento e inizi Novecento si diressero dalla montagna veneta verso i territori dell'Europa centro-orientale. Soprattutto il Bellunese fu interessato a partire dalla metà dell'Ottocento da una considerevole migrazione stagionale verso l'Europa centro-orientale, che, in inverno, trovò impiego nella ristrutturazione stradale e ferroviaria o nei lavori connessi al taglio delle piante. Nei mesi estivi se ne aggiunse, tuttavia, un'altra di venditori ambulanti di dolciumi, a cui subentrò in seguito la vendita del gelato. Tracce di entrambi i flussi si rinvengono nei racconti orali del mio campione che riferisce di una forma singolare e tuttora attiva di emigrazione stagionale. Praticata dal lontano 1880 da intere vallate dell'Alto Veneto, essa si rivolse inizialmente a Vienna e ai territori limitrofi dell'Impero asburgico e, subito dopo la II guerra mondiale, soprattutto alla Germania. Il materiale qui proposto, desunto dalla mia tesi di dottorato dal titolo I gelatieri veneti in Germania. Un'indagine sociolinguistica, pubblicata nel 2006 dalla Peter Lang di Francoforte sul Meno, si fonda su 
un'indagine quantitativa e qualitativa. Grazie alle testimonianze autentiche sarà, pertanto, possibile intraprendere un "viaggio" nella memoria di intere vallate montane, "consacrate" da secoli, all'emigrazione stagionale artigianale.

Parole chiave: Veneto, Europa centro-orientale, emigrazione stagionale, professioni storiche, memoria orale

\section{Introduzione}

Il presente contributo si occupa delle migrazioni stagionali che, tra fine Ottocento e inizi Novecento, dalla montagna veneta si rivolsero verso i territori dell'Europa centro-orientale, in particolare verso la "Germania", la "Romania" e i Balcani.

Inizialmente, si opererà una differenziazione tra l'emigrazione transoceanica e quella temporanea e stagionale dal Veneto. In seguito, verrà evidenziata l'importanza dei movimenti artigianali che dalla montagna veneta si diressero, con svariate specializzazioni, oltralpe. In particolare, si prenderanno in considerazione alcune professioni "storiche", come quelle degli "esamponari" o "squarador", che, in diversi paesi dell'Europa centro-orientale trovarono impiego, in inverno, nella ristrutturazione di strade e ferrovie ("esamponari") o nei lavori connessi al taglio delle piante ("squarador").

A questa ondata migratoria se ne associò, in estate, un'altra di venditori ambulanti di dolciumi, sostituiti, in seguito, dalla vendita del gelato. Ambedue i flussi vengono menzionati nelle memorie orali del campione da me intervistato che riferisce di una forma singolare e tuttora attiva di emigrazione stagionale. Praticata dal lontano 1880 da intere vallate dell'Alto Veneto, essa interessò inizialmente Vienna e i territori limitrofi dell'Impero asburgico e, subito dopo la II guerra mondiale, soprattutto la Germania.

Il materiale qui presentato è stato estrapolato dalla mia tesi di dottorato dal titolo I gelatieri veneti in Germania. Un'indagine sociolinguistica, pubblicata nel 2006 dalla Peter Lang di Francoforte sul 
Meno. Il suddetto lavoro si basa su un'indagine sia quantitativa, tramite la compilazione di 196 questionari (dei 300 somministrati), sia qualitativa grazie al ricorso a due tipologie d'intervista: "strutturata a risposta prefissata" e "semi-strutturata a risposta libera"[1].

L'apporto delle testimonianze orali ci permetterà, inoltre, di intraprendere un viaggio attraverso la memoria di intere vallate, contrassegnate da una secolare tradizione migratoria stagionale.

\section{Emigrazione definitiva e temporanea dal Veneto ${ }^{[2]}$}

Tra la fine dell'Ottocento e gli inizi del Novecento si assistette in Italia a un'emigrazione di massa di tipo definitivo e transoceanico: si calcola che tra il 1876 e il 1915 espatriarono più di quattordici milioni di italiani, con punte massime del $40 \%$ in alcuni anni dell'ultimo quindicennio del secolo XIX (cfr. Rosoli, 1978, 217).

Il contributo del Veneto, soprattutto delle province di Belluno e Treviso, fu molto significativo nel periodo tra il 1888 e il 1891, con un picco di espatri del 33,85\% nel decennio compreso tra il 1891 e il 1900 (cfr. Bottin, 2014, 138).

Tra il 1876 e il 1886, 54.745 persone, provenienti per lo più dalle zone prealpine e pedemontane, emigrarono verso le pianure meridionali brasiliane e argentine (cfr. Franzina, 1991, 49), mentre tra il 1887 al $1897 \mathrm{fu}$ la volta di intere famiglie delle pianure venete dirette verso le piantagioni di caffè del Brasile. In quel periodo le statistiche ufficiali rilevano circa 330.000 persone emigrate, pari a circa il 110 per mille della popolazione (cfr. Lazzarini, 1983, 22-24).

Condizioni economiche disagiate e povertà furono alla base dell'esodo definitivo, oltre alla "crisi profonda dell'agricoltura regionale", determinata dal "passaggio dall'economia di tipo precapitalistico [...] ad

[1] Si veda anche Campanale, 2006a, 21-27, così come Caldognetto e Campanale (a cura di), 2014, 123-140.

[2] Secondo Bottin $(2014,132)$, "l'antica tradizione delle popolazioni montane di effettuare spostamenti temporanei verso i paesi d'oltralpe con lo scopo di ottenere un reddito aggiuntivo a quello agricolo, aiutò senz'altro ad instillare nelle popolazioni della pianura l'idea che al di fuori della propria realtà esistessero condizioni migliori". Anche altri studiosi sostengono che "i network di mobilità del periodo dell'Impero Asburgico abbiano determinato una peculiare «cultura migratoria» che è sopravvissuta nel tempo, al punto da indirizzare anche i percorsi «forzati» degli spostamenti in massa successivi alla fine della Prima e della Seconda guerra mondiale, [...]". (Marzi, Alessio. Attraversati dai confini. Emigrare rimanendo (quasi) fermi. Disponibile da https://www.asei.eu/it/2017/03/attraversatidai-confini-emigrare-rimanendo-quasi-fermi/). 
una moderna economia capitalistica", in seguito all'annessione della regione all'Italia (Culatti, 1997, 25-27) ${ }^{[3]}$.

A ciò si aggiunsero "[1]e politiche economiche attuate prima dall'Impero asburgico e successivamente dal Regno d'Italia ${ }^{[4]}$, la crisi finanziaria con il crollo della Borsa di Vienna (1873) e l'introduzione di un regime di imposte fondiarie che penalizza[ndo] le piccole proprietà [...] minava il precario equilibrio di un'economia di sussistenza"[5].

Nonostante l'importanza dell'emigrazione definitiva oltreoceano, non bisogna dimenticare che, verso la fine dell'Ottocento, si registrarono anche considerevoli flussi, provenienti dal Veneto, che si orientarono temporaneamente verso le mete europee, più facilmente raggiungibili dai luoghi d'origine (cfr. Culatti, 1997, 16). Nel Bellunese ci furono 20.000 partenze temporanee negli ultimi anni del secolo, con un incremento di oltre 25.000 nel 1900 (cfr. Culatti, 1997, 36-37) ${ }^{[6]}$.

Tra il 1915 al 1920 i picchi più alti di partenze temporanee furono dirette soprattutto verso l'Impero austro-ungarico, la Svizzera, la Francia o la Germania, a causa delle scarse opportunità lavorative in patria e, per contro, delle maggiori retribuzioni degli operai nei paesi di accoglienza. Si trattava per lo più di "contadini, braccianti ed artigiani, specialmente [...] muratori e calderai" che partivano tra la fine dell'inverno e la primavera (cfr. Bernardi e Todisco, 1998, 20-21; 89-90).

Durante il Ministero di Giolitti l'emigrazione temporanea ed europea fu così importante che ne risentì, a livello civile e organizzativo, tutta la fisionomia della montagna, mentre con l'avvento del Fascismo (1920-1930) si determinò un arresto dell'emigrazione verso l'estero dal Bellunese e dal Feltrino con notevoli ripercussioni economiche negative per le suddette aeree (cfr. Franzina, 1991, 211; 222).

[3] Non è casuale che furono proprio i contadini, mezzadri, piccoli fittavoli veneti a lasciare definitivamente il paese per dirigersi in Sud America, con il miraggio di diventare proprietari terrieri (cfr. Franzina, 1991, 49).

[4] "Con lo scoppio della Grande Depressione (1873-96) in tutta Europa si verifica un cambio delle politiche economiche nazionali. Il continuo abbassamento dei prezzi agricoli comporta un rialzo delle imposte doganali [...], ma la vera politica protezionistica si ha con la tariffa del 1888 che pone tasse sempre più alte a quasi tutti i tipi di merci che sono importate nel regno" (Bottin, 2014, 6).

[5] http://emigrazionetrentina.museostorico.it/la-grande-emigrazione.

[6] Si stima che nelle province di Belluno, Udine e Vicenza l'emigrazione temporanea rappresenti circa l's8\% di quella regionale (cfr. Bottin, 2014, 135). 


\section{L'emigrazione stagionale specializzata dalla montagna veneta $^{[7]}$}

Da secoli, l'intero versante alpino sarebbe stato contraddistinto da un'emigrazione stagionale di artigiani e ambulanti. Tale tipologia migratoria, presente anche nel Bellunese, fu caratteristica di alcuni territori confinanti dell'arco alpino, come il Ticino, il Tirolo, la Baviera, la Valsugana ${ }^{[8]}$. Essa sarebbe stata determinata da fattori negativi come "la sovrappopolazione delle Alpi, clima ingrato, raccolti distrutti, incendi, epidemie, eccedenza delle nascite, miseria stabile" (Burmeister, 1994, 13), ma anche positivi quali "l'ambizione" e "la prospettiva di guadagni materiali, facilitati p. es. da forte espansione edilizia in certe regioni" (Burmeister, 1994, 13).

Allo stesso tempo, si possono rinvenire alcuni aspetti comuni, quali il carattere "stagionale trasversale" (Ceschi, 1994, 22) e il fatto che si trattasse essenzialmente "di un'emigrazione di servizi e di commerci" o "girovaga" (Ceschi, 1994, 16). Inoltre, a fine Ottocento, si aggiunse, al flusso migratorio maschile, la componente femminile e minorile (cfr. Palme, 1994, 251; 253), anch'essa impiegata nei lavori di ristrutturazione delle vie di comunicazione, in particolare di quelle stradali e ferroviarie (cfr. Palme, 1994, 252).

Da non dimenticare, inoltre, la presenza "di una rete informale che collegava il luogo di partenza a quello di destinazione, basata sulle interrelazioni all'interno del gruppo familiare e del gruppo locale" (Grandi, 1994, 298), nonché il carattere di necessaria complementarità, in quanto, "coincide[ndo] con i periodi di punta delle attività agricole, [andava a] colmare i troppi vuoti lasciati da un esodo massiccio" (Ceschi, 1994, 27) della popolazione locale, emigrata a sua volta stagionalmente in cerca di maggiori guadagni:

L'emigrazione degli alpigiani oltre il confine era un fatto consolidato [...] perché era un fenomeno insito nella struttura economica delle Alpi, [...]. [A] partire dal XVII secolo nelle zone

[7] Cfr. Campanale, 2006a, 33-36.

[8] I kròmeri (dal tedesco kramer), merciai ambulanti che provenivano dalla Valsugana e dal Tesino "praticavano un'emigrazione stagionale a corto raggio recandosi in pianura Padana, Tirolo, Baviera e Svizzera” (http:// emigrazionetrentina.museostorico.it/la-grande-emigrazione). 
alpine l'emigrazione, ed in particolare quella stagionale, diventa un «fattore strutturale» della stessa economia delle Alpi a causa delle caratteristiche morfologiche-geografiche e della struttura economica più arretrata rispetto alle confinanti aree di pianura (Grandi, 2007, 77) ${ }^{[9]}$.

\section{Come dalle confinanti regioni del Trentino ${ }^{[10]}$ e del Friuli ${ }^{[11]}$, anche} dalle località montane del Bellunese, si sarebbero spostate, già in epoca preindustriale, correnti costanti di artigiani ambulanti, specializzati in determinati e specifici settori di attività, per dirigersi verso le città, prima

[9] Anche Bovenkerk e Ruland (1992, 927; 934 ss.) ritengono che l'emigrazione artigianale stagionale alpigiana che si protrasse fino al XX secolo, sarebbe scaturita come "risposta alla crescita demografica e all'insicurezza economica nelle regioni montane di provenienza [, per cui] la specializzazione del commercio e del lavoro artigianale nacque come soluzione alternativa ai magri proventi di un'agricoltura alpina e alle altre poche alternative industriali, praticate nei mesi invernali. Si sarebbe trattato di un sistema circolare d'emigrazione a catena, in cui gli abitanti dello stesso paese che si specializzavano in una determinata arte o mestiere, emigravano all'estero in una città che non era ancora stata occupata dalla stessa categoria, richiamando poi a catena personale che proveniva dalla stessa famiglia o dallo stesso paese" (Campanale, 2006a, 113).

[10] Anche nel vicino Trentino, le correnti migratorie stagionali di artigiani, iniziate nel Settecento "proseguirono nel XIX secolo ampliando il proprio raggio di azione e la gamma dei prodotti trattati. I perteganti (venditori ambulanti del Tesino) si specializzarono nel commercio di stoffe e strumenti ottici, dirigendosi in tutta Europa. I parolòti (venditori e riparatori di utensili in metallo della val di Sole) viaggiavano invece verso sud lungo l'Italia, ma la loro presenza è attestata anche in Francia. Gli spazzacamini, che partivano soprattutto dalla bassa valle di Non e dal Banale, e i segantini delle Giudicarie trovarono le migliori opportunità di lavoro nell'Italia settentrionale. Tra il 1880 e la Prima guerra mondiale si ebbe il periodo di maggiore sviluppo e successo commerciale dei moléta, ossia gli arrotini. La maggior parte di questi lavoratori proveniva dalla val Rendena. [...] L'Ottocento vide anche emergere professioni nuove come quella dei careghéta (seggiolai) e dei kròmeri, merciai ambulanti. I primi, costruttori e riparatori di sedie, dal Primiero e da Belluno si spostavano nelle pianure limitrofe restando lontani da casa da ottobre ad aprile. Gradualmente i loro percorsi si fecero più lunghi andando a raggiungere la Francia, la Svizzera, il Belgio, il Lussemburgo e tutto l'Impero austro-ungarico. Tutte queste professioni (ad eccezione degli arrotini), che riunivano aspetti artigianali e commerciali e che si occupavano della riparazione degli oggetti, sparirono all'inizio del Novecento poiché rese obsolete dalla più economica produzione industriale" (http://emigrazionetrentina.museostorico.it/lagrande-emigrazione).

[11] Nel Friuli-Venezia Giulia "il movimento pendolare e migratorio a corto raggio che connetteva i territori limitrofi raggiunse i suoi massimi sviluppi [dopo l’Unità d'Italia]. Per i friulani, [...], lo spostamento temporaneo, ripetuto o stagionale nei territori rimasti sotto il dominio degli Asburgo continuò a rappresentare una risorsa economica importante anche dopo il 1866, nonché un vero e proprio «stile di vita», lo stesso che fin dall'Ancien Régime aveva caratterizzato la storia dei «cramars», i venditori ambulanti che partendo dalle zone di montagna, si spingevano per la propria attività commerciale nei territori a valle di entrambi i versanti dell'arco alpino, smerciando anche i prodotti dell'industria tessile ed alimentare domestica della Carnia. A partire dagli anni 1880, le possibilità occupazionali e le mete del lavoro temporaneo o stagionale in Austria-Ungheria per gli operai friulani di collina o di montagna addirittura si moltiplicarono grazie agli sviluppi dei mezzi di trasporto e di comunicazione. A partire da Trieste o dalla vicina Carinzia, [...], i friulani scoprirono e sfruttarono possibilità di lavoro e guadagno in Germania, in Slovenia, in Ungheria, in Romania, in Bosnia, in Serbia, [...]. L'intera area danubiana e mitteleuropea diventò quindi, nonostante il confine, uno spazio aperto, familiare e quindi raggiungibile, [...], [in cui] [...] i tradizionali network di lavoro transfrontaliero, da locali ed a corto raggio, divennero [...] «continentali», [...].[...] È bene ribadire che, a contrario dell'emigrazione diretta verso Francia e Svizzera, l'emigrazione italiana in AustriaUngheria rimaneva, anche a cavallo di Ottocento e Novecento, prevalentemente emigrazione temporanea, veneta e friulana, quindi, in senso lato, «frontaliera»; vedeva cioè protagoniste soprattutto, [...], le popolazioni vicine al confine. [...] Giovanni Cosattini della Società «Umanitaria», riteneva che all'inizio del Novecento il Friuli fornisse circa un quarto di tutta l'emigrazione temporanea italiana che stimava nel 1903 in circa 280.000 persone. [...]. Tale «sistema migratorio» crollò allo scoppio della Prima Guerra Mondiale, [...]; tuttavia, negli anni successivi al conflitto, il dirottamento dell'emigrazione temporanea friulana verso altre mete europee (principalmente Belgio, Francia e Svizzera) o l'Africa Orientale Italiana fu letto e vissuto come espressione «alternativa» dei tradizionali spostamenti frontalieri, pendolari o stagionali per lavoro verso Trieste, l'Austria-Ungheria e la Germania" (Marzi, op. cit.). 
italiane, e poi straniere, tanto che ogni valle avrebbe avuto una determinata qualificazione (cfr. Culatti, 1997 e Brunold, 1994). Dall'Agordino, partivano facchini, muratori, fabbri, seggiolai ${ }^{[12]}$, cioccolatai, salsicceri; dal Comelico gli stagnini; da Sappada imbianchini e decoratori; dallo Zoldano, dagli inizi dell'Ottocento fino agli anni 1930, emigrarono a Milano gli Scòti, venditori ambulanti di caldarroste, pere cotte, dolci, frutta caramellata; da Selva, verso la laguna veneta, pasticceri e salumai e infine dall'Alpago cuochi, domestiche e balie (cfr. Culatti, 1997, 35).

Dalla metà dell'Ottocento questo sistema basato sulla pratica sistematica dell'emigrazione stagionale iniziò ad incrinarsi per effetto di forti fattori attrattivi, come l'ingente richiesta di manodopera nei paesi dell'Europa centrale.

L'emigrazione "degli alpigiani", oltre a garantire per secoli la sopravvivenza di intere vallate, fu fondamentale per lo sviluppo di successivi modelli migratori, poiché:

spianò la strada, nel Veneto, anche [...] agli esodi da lavoro prodottisi nella regione a far data dagli anni '50 e in seguito all'avvio delle grandi imprese edilizie, stradali e minerarie che allora si ebbero su scala italiana e ancor più europea. [...] Esso avrebbe condizionato a lungo le sorti di una parte cospicua dell'emigrazione veneta di tipo temporaneo, rinnovandosi, e rinnovandola, sin dentro il secolo successivo, come accadde ad esempio quando, fra il 1883 e il 1888, una media di quattromila lavoratori bellunesi e friulani, trevigiani e vicentini, accorsi in Serbia «ai lavori», costrinse le nostre autorità ad «istituire a Nisch un viceconsolato» (Franzina, 1991, 56).

[12] I seggiolai, definiti nel dialetto locale karegete e nel gergo della loro categoria konža provenivano dall'Agordino, in provincia di Belluno. "[Il] mestiere che sembra essere nato a Gosaldo (BL), in particolare nella frazione di Tiser tra il 1780 e il 1790, per poi spargersi in tutto l'Agordino, e nei paesi di Sagron e Mis nel Trentino [, d] ate le condizioni di miseria in cui riversava la vallata, [...] rappresentava una valida alternativa ad una emigrazione definitiva o al duro lavoro in miniera. [...] [D]al giorno di San Rocco (16 agosto) cominciavano le partenze dei karegete, che in genere ritornavano in primavera, per i lavori agricoli. [...] I seggiolai coprivano tutte le regioni settentrionali dell'Italia, e scendevano fino anche in Abruzzo e in Lazio; inoltre gran parte degli emigranti trovava lavoro in Francia e qualcuno anche in Svizzera, Belgio e Lussemburgo. [...] Il gruppo di lavoro era formato dal «maestro artigiano» [...] e da uno o più garzoni". [...]. È facile intuire come il mestiere del karegeta, essendo sempre stato inteso come un arrangiamento provvisorio, [...], con l'industrializzazione del secondo dopoguerra sia rapidamente scomparso" (Broch, 2010, 5-7). 


\section{L'emigrazione italiana verso la Germania e il contributo veneto $^{[13]}$}

Già dal XIII secolo gli italiani erano presenti in Germania come banchieri, gabellieri o direttori di zecca, mentre nel XVII e XVIII secolo erano conosciuti come educatori, musici, cantanti, teatranti, commercianti (importatori, spazzacamini, venditori ambulanti), ma anche come architetti, pittori, stuccatori, scultori (cfr. Bade, 1992, 288).

Dal XVII ai primi venti anni del XX secolo, l'emigrazione italiana era fortemente specializzata (come nel caso dei terrazzieri friulani o degli stuccatori comaschi) e legata alla provenienza, per lo più dalle zone montane del Piemonte, della Lombardia, del Veneto e del Friuli (cfr. Pilcher, 1992, 8).

A partire dall'ultimo quinquennio del XIX secolo, i nostri connazionali si diressero numerosi verso l'Impero tedesco ${ }^{[14]}$, trovando impiego nell'industria edile e nelle fornaci. Provenivano per lo più dalle regioni settentrionali (il 50\% dal Veneto) e si trasferirono, data la vicinanza, soprattutto verso l'Austria-Ungheria ${ }^{[15]}$, la Germania meridionale e sudoccidentale $^{[16]}$ (in Baviera, nel Württemberg, nel Baden e in AlsaziaLorena), ma anche nelle due province prussiane occidentali della Renania e della Vestfalia (cfr. Del Fabbro, 1993, 28-30):

I territori, che appartenevano alla Germania solo dal 1871, attirarono l'immigrazione italiana principalmente per la vicinanza geografica al paese d'origine e per le propizie possibilità di occupazione, conseguenti ad un'ampia offerta di posti di lavoro in ambito industriale nei settori in rapida espansione del lorenese, ma anche per la legislazione per gli stranieri di carattere liberale. [...] Mentre in Prussia si sviluppò ben presto, come reazione all'afflusso in massa di manodopera polacca, una legislazione per gli stranieri diversificata, la

[13] Cfr. Campanale, 2006a, 27-32.

[14] Il flusso migratorio fu sempre più consistente: da 4.000 persone nel 1871 a 170.000 nel 1913. (cfr. Del Fabbro, 1993, 28-30 e Campanale, 2006a, 28).

[15] Per quanto riguarda il Veneto, il flusso emigratorio era diretto principalmente verso l'Impero asburgico - date la vicinanza e la possibilità di accedervi senza passaporto -, da qui è probabile che molti lavoratori transitassero dall'Austria verso la vicina Germania alla ricerca di un'occupazione adeguata (cfr. Grandi, 2001, 361).

[16] Gli stati sudoccidentali attirarono maggiormente la manodopera italiana, sia per la "vicinanza geografica" sia per la mentalità "più affine alla cultura latina rispetto a quelle di altre zone dell'Impero tedesco" (Trincia, 2001, 250). 
Baviera, il Württemberg, il Baden e l'Alsazia-Lorena si opposero con successo, fino alla fine dell'età imperiale, contro un regolamento di modello prussiano [...]. Non vennero introdotte, pertanto, in tali regioni né l'obbligo di legittimazione (la registrazione obbligata di una persona attraverso il rilascio di una carta d'identità al lavoratore), né il periodo di carenza (l'obbligo di lasciare il paese durante i mesi invernali). [...] $\mathrm{Al}$ più tardi nel secondo quinquennio del $\mathrm{XX}$ secolo si era, pertanto, cristallizzato "un mercato del lavoro straniero diviso»: il repressivo regno prussiano da una parte e, dall'altra, gli Stati del Sud della Germania che adottavano una procedura libera. [...]. Gli italiani trovarono occupazione quasi esclusivamente nell'industria [...], soprattutto nell'industria edile e nelle fornaci, diventati i settori d'occupazione privilegiati dagli italiani, anche per via del loro carattere stagionale. Parecchi autori del tempo vedevano nei lavoratori italiani dell'impero un potenziale di manodopera di seconda classe rispetto al proletariato locale [...], [dato che la maggior parte era rappresentata da] lavoratori «non qualificati» (Del Fabbro, 1993, 30-33).

Di solito, gli italiani emigravano "sotto la direzione di un intermediario, cioè di un caposquadra italiano, che sceglieva in patria un certo numero di lavoratori che gli sembravano adatti al lavoro da svolgere in Germania, dove venivano impiegati come «squadra» per lo più stagionalmente" (Del Fabbro, 1993, 39). Alcuni con il tempo divennero imprenditori e piccoli commercianti, nei settori che "comprendevano particolari professioni, che usufruivano di una lunga tradizione in Italia e la cui richiesta in Germania, a causa dello sviluppo dell'edilizia, era in continua crescita, come, per esempio, i famosi «terrazzai» veneziani, che producevano pavimenti a mosaico di pietre e cemento" (Del Fabbro, 1993, $35)$.

Agli inizi del Novecento, l'emigrazione italiana, da stagionale, divenne temporanea su base "pluriennale" e spesso definitiva, mentre con l'inizio della Grande Guerra il numero di presenze scese drasticamente, tanto che alla fine del 1924 c'erano in Germania solo circa 21.900 italiani (cfr. Del Fabbro, 1993, 42-43).

Per quanto riguarda i veneti, il flusso temporaneo come sterratori o persone dedite alle industrie ambulanti ${ }^{[17]}$ era rivolto principalmente verso

[17] In Germania nel 1881 c'erano 5.558 emigrati, di cui quasi tutti, cioè 5.059, provenienti dal Veneto, in 
la Germania meridionale (Württemberg e Baviera). Nel 1883 si registravano nel Württemberg circa 400 italiani con migrazione temporanea (rimpatrio in inverno, ritorno in primavera), impiegati come braccianti, muratori, scalpellini, calderai, suonatori e venditori di frutta, mentre in Baviera, negli anni 1884, ci sarebbero stati circa 700 nostri connazionali occupati come fornaciai, muratori, minatori, tagliapietre, calderai, filatori di cotone, fruttivendoli e stuccatori (cfr. Bernardi e Todisco, 1998, 68-69) ${ }^{[18]}$.

\section{L'emigrazione italiana verso la Romania e il contributo del Veneto ${ }^{[19]}$}

Gli italiani iniziarono ad emigrare in Romania nell'Ottocento, trovando impiego come manovalanza non specializzata, dedita alla pastorizia o all'agricoltura ${ }^{[20]}$ (cfr. Pirvu, 2018, 636). La maggior parte si stabilì nel 1860 nel Sud-Est del Paese, come tagliatori di pietra o lavoratori edili. Nei Monti Carpazi gli italiani costruirono importanti opere, come la stazione ferroviaria, ma anche monumenti funerari per le grandi famiglie nobiliari rumene a Bucarest, Iassy e Targoviste ${ }^{[21]}$ (cfr. Pirvu, 2018, 640).

In seguito, i nostri connazionali trovarono lavoro in tutto il territorio rumeno ${ }^{[22]}$, come manodopera qualificata nelle miniere, ma soprattutto nell'edilizia-come architetti-(cfr. Scagno, 2008, 107-108) e nelle infrastrutture, come manovali, muratori, squadratori, scalpellini, marmisti, falegnami, fabbri, fornaciai, contribuendo alla realizzazione di costruzioni sanitarie, infrastrutture, strade, chiese, teatri, ponti, scuole, e insegnando ai rumeni il mestiere ${ }^{[23]}$ (cfr. Scagno, 2008, 24-27 e Pirvu, 2018, 636).

particolare dalle province di Udine e Belluno. Si trattava per lo più di "terraiuoli” e muratori veneti stagionali. I residenti nel 1878 erano circa 200, impiegati in banche o agenzie commerciali o come muratori-carpentieri. In qualità di emigranti specializzati si trovavano poi "fabbricanti di figure di gesso", "stuccatori, scultori di rilievi, lavoratori di pavimenti alla veneziana ed ebanisti” (Grandi, 2001, 364-365).

[18] Anche dopo la seconda guerra mondiale continuerà l'afflusso di veneti in Germania: si tratterà per lo più di stagionali, impiegati nelle gelaterie, provenienti soprattutto dalle province di Belluno e Treviso. Nel 1986 si calcola che di 5.071 veneti, 3.304 fossero della provincia di Belluno e 1.498 di Treviso (cfr. Pilcher, 1991, 13).

[19] Cfr. Campanale, 2019, 259-274.

[20] Cfr. anche Di Cesare, Valentina. La comunità italiana in Romania. Disponibile da http://www.lundici. it/2016/05/emigrazione-la-comunita-italiana-inromania/ (consultato il 09 febbraio 2020).

[21] Cfr. Rodica Chiretu, Marilena. Emigrazione italiana in Romania. Disponibile da https://www.pitestiromania. ro.

[22] Fino al 1881, in mancanza di controlli ufficiali da parte delle autorità rumene, si trattava di un'emigrazione essenzialmente temporanea e stagionale (cfr. Scagno, 2008, 23).

[23] Cfr. Di Cesare, Valentina, op. cit. 
Tra il 1866 e il 1881, la Romania iniziò un forte processo di modernizzazione, per cui diede "in concessione a società straniere austriache, inglesi e tedesche la creazione dell'intero sistema ferroviario nazionale [...], [ma anche] della rete stradale distrettuale e dell'assetto urbano delle principali città" (Scagno, 2008, 24-25; 27). Tali società appaltavano e subappaltavano i lavori ad imprese ${ }^{[24]}$ che si servivano a loro volta di intermediari che reclutavano i lavoratori stranieri nei paesi d'origine, per lo più nella Transilvania e nella Bucovina austriache, ma anche in Bulgaria, in Serbia e spesso nell'Italia nord-orientale (nel Veneto, Friuli e Trentino). Gli emigrati venivano impiegati nel settore ferroviario, a livello di manovali, muratori, ma persino fabbri e falegnami (cfr. Scagno, 2008, 25-26).

Alla base del notevole afflusso di manodopera italiana qualificata (soprattutto veneta) alla fine dell'Ottocento, “[l'] aggravarsi della crisi economica e sociale italiana di quegli anni, [...] [1'] inizio della grande emigrazione transoceanica, ma anche [il] consolidarsi dei rapporti diplomatici tra i due Paesi" (Scagno, 2008, 37).

Dal 1871 al 1901 i flussi migratori dall'Italia verso la Romania furono sempre più consistenti: da 870 a 8000 persone fino ad arrivare a 60.000 nel 1930 (cfr. Pirvu, 2018, 637) ${ }^{[25]}$, per cui, in seguito alle proteste degli operai rumeni contro gli stranieri, fu varata la cosiddetta "legge dei mestieri", che imponeva di assumere con precedenza i rumeni.

Il 15 agosto 1900 venne, inoltre, emanato un regolamento "protezionista", secondo il quale gli stranieri, oltre l'ottavo giorno di permanenza in Romania, sarebbero stati costretti a richiedere alle prefetture un permesso di soggiorno ("biglietto di libero soggiorno"), "presentando un passaporto rilasciatogli dalle competenti autorità dello Stato di appartenenza e previamente vistato dalla [propria] [...] legazione o consolato in Romania" (Scagno, 2008, 42).

Nel 2015, lo scoppio della Grande Guerra comporterà un'interruzione dell'emigrazione temporanea in Romania, che riprenderà

[24] In poco tempo gli imprenditori italiani si distinsero tanto da essere al primo posto tra le società appaltatrici straniere nel ramo dell'edilizia pubblica e privata (cfr. Scagno, 2008, 36).

[25] Nel 1912, circa 6.500 italiani risiedevano temporaneamente in Romania, nel 1927 ce n'erano persino 12.246, di cui 8.000 nel solo Distretto consolare di Bucarest. Diversi i permanenti che esercitavano svariate professioni in molteplici settori, dal primario al terziario, e che si distinguevano per l'alto grado di specializzazione, ma anche per la loro onestà e laboriosità. Tra le colonie fisse troviamo, ancora una volta, soprattutto veneti e friulani, ma anche rappresentanti di altre regioni italiane (Scagno, 2008, 58-60). 
subito dopo, cioè tra il 1919 e il 1925, con “542 riservisti e 177 famigliari” e 720 con un contratto di lavoro (Scagno, 2008, 54-56).

Poc'anzi, abbiamo visto, che tra la fine dell'Ottocento e gli inizi del Novecento, il "sogno americano" fu rappresentato dalla Romania-in particolare dalla Transilvania-, soprannominata "la California romena" ${ }^{[26],}$ in cui gli stipendi erano molto più alti di quelli italiani ${ }^{[27]}$. Ciò portò migliaia di famiglie, soprattutto venete e friulane, a trasferirsi nel Sud-Est del paese, per lo più nella regione di Craiova ${ }^{[28]}$.

Alla fine del XIX secolo ${ }^{[29]}$, circa il 10-15\% dei veneti sarebbe emigrato stagionalmente o temporaneamente in Romania, trovando impiego nell'edilizia, nella costruzione delle ferrovie, in attività boschive ${ }^{[30]}$ o nelle miniere ${ }^{[31]}$, spesso sulla base del passaparola tra compaesani:

L'emigrazione dal Veneto aveva assunto un carattere differente da quello del ventennio precedente quando i lavoratori venivano assunti collettivamente da impresari stranieri o per mezzo di loro intermediari in Italia. [...]; si sviluppò quindi, anche attraverso il passaparola con i compaesani e i valligiani delle località di origine, una forma di emigrazione individuale o a piccoli gruppi guidati da capomastri locali o alle dipendenze di subappaltatori, e sempre più sovente venne scelta la forma del lavoro a cottimo (Scagno, 2008, 34-35).

Nel 1924, a causa di un regolamento ancora più restrittivo sul controllo dei lavoratori stranieri si concluderà la stagione dell'“emigrazione temporanea dalle province venete come fenomeno stagionale collettivo", senza comunque causare "la scomparsa della presenza veneta e friulana nell'attività edilizia urbana pubblica e privata" (Scagno, 2008, 57).

Tra gli anni Venti e lo scoppio della Seconda Guerra Mondiale, in molte città e cittadine delle diverse province rumene, si registra, infatti,

[26] Cfr. Raluca Torre, Andreea. La migrazione italiana in Romania. Etnografia di un villaggio della Dobrugia. Disponibile da https://www.emigrazione-notizie.org/.

[27] Cfr. Baciu, Mirela. Quando i, rumeni' erano gli italiani. Disponibile da http://www.blog2fete.com/quando-irumeni-erano-gli-italiani, così come Scagno, 2008, 34.

[28] Cfr. Di Cesare, Valentina, op. cit.

[29] Cfr. Caritas Italiana, 2008, 59-61, così come Scagno, 2008, 34.

[30] Secondo le fonti, alcune famiglie dell'odierno Triveneto furono condotte nel 1821 in Transilvania, a lavorare come tagliaboschi e lavoratori del legno per conto di un commerciante austriaco di legname, in quanto "[l]'Austria Ungheria tende[va] a favorire le migrazioni interne tra le regioni più povere e di confine" (Caritas Italiana, 2008, 59).

[31] Cfr. Bontempelli, Sergio. Quando gli italiani emigravano in Romania. Disponibile da http://www. sergiobontempelli.wordpress.com/2008/09/07/italianiinromania/. 
sia la presenza di alcuni famosi impresari, costruttori e architetti veneti e friulani, sia di artigiani specializzati, come "mosaicisti, marmisti, decoratori, stuccatori, incisori e modellatori di lapidi e pietre sepolcrali, ebanisti, [...] particolarmente apprezzati in settori artigianali non coperti da maestranze qualificate romene [...]" (Scagno, 2008, 58).

Tra la fine dell'Ottocento e gli inizi del Novecento, il ruolo e il prestigio acquisito dagli emigrati stagionali veneti ne avevano determinato una trasformazione e stratificazione sociale ed economica all'interno della società romena (Scagno, 2008, 48):

Lavoratori tenaci e intraprendenti avevano posto le basi di attività imprenditoriali stabili nel Paese, con l'intento di metterle a frutto anche nel settore dell'edilizia privata che presentava grandi potenzialità di sviluppo con l'incremento della necessaria modernizzazione delle strutture urbane. I muratori e gli scalpellini erano sempre i più ricercati e i più apprezzati per «continuità del lavoro» e per «abilità tecnica», riuscendo ad ottenere salari più alti di quelli dei loro colleghi romeni e stranieri (Scagno, 2008, 48-49).

Con il fascismo, la Seconda Guerra Mondiale e soprattutto il regime comunista di Ceauşescu anche l'emigrazione veneta verso la Romania si arrestò, ma riprese subito dopo la caduta della Repubblica Socialista di Romania, in virtù dei secolari legami tra il Nord Est dell'Italia e l'Europa centrale, supportata dai costi contenuti della manodopera e dalle opportunità di un mercato in piena espansione ${ }^{[32]}$.

\section{L'emigrazione veneta verso i Balcani}

I rapporti tra i Balcani e il Veneto, in particolare tra la Repubblica della Serenissima e i territori che si affacciano sull'Alto Adriatico (Istria, Croazia, Dalmazia ecc.), sono datati e risalgono al lontano Medioevo:

In età basso-medievale, tra i secc. X e XV, gli influssi dello spazio linguistico italiano sull'area balcanica interessano - grazie soprattutto al ruolo di Venezia, emergente potenza marinara

[32] Cfr. De Matteo, Lynda. La Corsa verso la Romania degli imprenditori italiani. Disponibile da https://www. institutdelors.eu $>2018 / 01>$. 
[...] - tutta la costa adriatica (dall'Istria alla Dalmazia: a Zara, a Spalato, a Dubrovnik/Ragusa, ecc.), quindi l'Albania, buona parte delle isole greche (Eptaneso e alcune Cicladi); quindi, segmenti strategicamente importanti del territorio della Grecia storica e, ancora, i grandi scali del Bosforo e del Pontos Euxinos $^{[33]}$.

Fino a tutto il XVIII secolo Venezia svolse, pertanto, un ruolo decisivo a livello economico, ma anche linguistico-culturale, in buona parte dell'area balcanica ${ }^{[34]}$, che tra il XVIII e il XIX secolo, passò sotto il dominio dell'Impero Asburgico ${ }^{[35]}$.

Alla fine dell'Ottocento, dopo l'Unità d'Italia, data la drammatica situazione italiana già più volte menzionata ${ }^{[36]}$, ci fu un notevole flusso migratorio dalle regioni nordorientali della nostra penisola, soprattutto dal Friuli e dal Veneto, verso l'attuale Bosnia, Croazia, Slovenia e Dalmazia, tanto che in quel periodo "fornaciai e muratori d[e]l Triveneto detenevano un vero «monopolio» lavorativo in Croazia" ${ }^{[37]}$, contribuendo alla rimodernizzazione delle città in cui si insediarono:

I primi arrivi di friulani e veneti nella città di Fiume risalgono ad epoche antiche, ma a cavallo tra Ottocento e Novecento [...] [essi] re[sero] possibile il rinnovamento edilizio della città e la costruzione di opere portuali. Vediamo una cospicua presenza dei nostri corregionali anche a Lubiana: dopo il terremoto nel 1895 i muratori svolsero un ruolo essenziale nella ricostruzione della città. E ancora troviamo manodopera friulana e veneta a Zagabria: dopo il terremoto del 1880 la città cambiò volto, e da vecchio centro provinciale austriaco si trasformò in una moderna città. Molti italiani risiedevano in altri centri, ad esempio Karlovac, Vinkovci e Sisak. Quest'ultima città, all'epoca, era un importante porto fluviale in cui la comunità friulana aveva

[33] Banfi, Emanuele. L'influsso dello spazio linguistico italiano sull'area balcanica. Disponibile da http:// brunodam.blog.kataweb.it/category/popoli-e-politiche/page/3/.

[34] Ibidem.

[35] Cfr. https://it.m.wikipedia.org/wiki/Slavonia.

[36] “[S]ubito dopo l'annessione del Veneto all’Italia dopo il plebiscito-truffa del 1866 [, i]l Regno d’Italia, [...], dopo le ingenti spese militari sostenute per la III guerra d'indipendenza si trovò sull'orlo della bancarotta; da qui la necessità di imporre una serie di tasse pesantissime, a partire da quella sul macinato che portò in breve la nostra terra veneta a una situazione di fame, miseria e disperazione come mai nella nostra storia: alla nostra gente non restò che emigrare, la stragrande maggioranza verso il Brasile, altri verso destinazioni diverse" (https://www.trevisotoday.it/cronaca/beggiato-emigranti-slavonia-novembre-2017.html).

[37] Cfr. Emigrazione italiana nei territori settentrionali dell'ex-Jugoslavia. Disponibile da http://brunodam.blog. kataweb.it/ category/popoli-e-politiche/page/3/. 
avviato il commercio di legname e la produzione di mattoni ${ }^{[38]}$.

In Croazia gli italiani erano impiegati come fornaciai, muratori, operai addetti alla realizzazione delle principali linee ferroviarie, capomastri, ma anche come impresari edili. Nel 1864 Andrea Colussi "trasformò il volto edilizio di Sisak: ristrutturò le vecchie opere fluviali, lastricò le strade, eresse palazzi pubblici ed abitazioni private"[39].

Sempre alla fine del XIX secolo, troviamo, inoltre, diverse presenze italiane in Slavonia ${ }^{[40]}$, a quel tempo sotto il dominio asburgico.

Si trattava di circa una novantina di famiglie provenienti dalle zone montane e pedemontane del Veneto e del Friuli che verso il 1880 si insediarono soprattutto nei centri rurali di Kutina, Lipik, Ploštine ${ }^{[41]}$, disboscando vaste aree per poter costruire le loro case e coltivare i campi ${ }^{[42]}$ :

Più della metà delle famiglie partirono dal Bellunese, in particolare dalla zona di Longarone, ma anche dalla provincia di Treviso (Conegliano, Orsago, Cordignano, Villa di Cordignano, Godega S. Urbano, Villorba), dall'Altopiano dei Sette Comuni (Asiago e Camporovere), dalla provincia di Pordenone (Sacile, Erto). Partirono a piedi, una lunga fila di carretti a due ruote, e arrivarono a destinazione dopo oltre un mese di marcia, assegnati a diversi paesi, Kutina, Plostine, Lipik, Pakrac e altri

[38] Ibidem.

[39] Ibidem.

[40] "La Slavonia, oggi in Croazia, è la zona compresa tra i fiumi Sava e Drava, affluenti del Danubio. È ricca di boschi, di riserve minerarie, di terra fertile e di acqua. I centri maggiori sono Pakrac e Lipik. [...] Nel corso del XVIII secolo il territorio venne spartito fra i nobili latifondisti locali; la famiglia dei conti Jankovic diventò proprietaria di uno dei poderi più vasti e lo gestì in maniere efficiente e innovativa. Si cominciò a disboscare e sempre più terreni vennero adibiti alla produzione agricola. Si sviluppò l'artigianato e il commercio, in particolare a Pakrac; su invito dei nobili e dei latifondisti cominciarono ad arrivare artigiani da tutta Europa grazie agli incentivi e alle agevolazioni offerte, come la possibilità di acquistare a rate case confortevoli con terreno. A Lipik si cominciarono a costruire stabilimenti per ripristinare l'uso delle acque termali a scopi curativi. Il XIX secolo fu il periodo di massimo sviluppo socio economico della Slavonia soprattutto per le iniziative e i finanziamenti proprio della famiglia Jankovic, in particolare del conte Izidor. A Lipik vennero completate e entrarono in funzione le strutture termali, accoglienti e funzionali tanto da diventare, verso la metà del secolo, un richiamo per tutta la nobiltà europea. Il conte Izidor investì anche sull'allevamento del bestiame, in particolare di cavalli di razza. Il successore, il conte Julie Jankovic, ereditò dal padre Izidor un patrimonio aggravato da pesanti debiti. Cercò di rimediare vendendo l'abbondante legname che la Slavonia offriva" (Barzan, 2014, 56-57).

[41] A Plostina, ad un centinaio di chilometri a sud di Zagabria, emigrarono verso il 1880 quarantacinque famiglie bellunesi, di cui "venticinque provenivano dalla zona compresa fra i comuni di Longarone, Castellavazzo, Soverzene, Ponte nelle Alpi e il paesino di Casso, nel comune di Erto in Friuli Venezia Giulia" (Barzan, 2014, 54). Ancora oggi, dopo quasi 150 anni, i discendenti di quei veneti vivono tuttora a Plostine, conservando gelosamente tradizioni, lingua, costumi della propria terra e restando ancorati alla loro identità veneta (cfr. https://www.bellunesinelmondo.it/tag/plostine/ così come https://corrierealpi.gelocal.it/tempolibero/2017/06/06/news/viaggio-a-plostine-da-150-anni-enclave-bellunese-1.15455361).

[42] Cfr. https://corrierealpi.gelocal.it/tempo-libero/2017/06/06/news/viaggio-a-plostine-da-150-anni-enclavebellunese-1.15455361. 
$\operatorname{ancora}^{[43]}$.

Alla base dell'esodo italiano, la situazione "favorevole" che si venne a creare in Slavonia nel 1876, quando le parti più estese dei territori della famiglia nobile Jankovic, gravate da pesanti debiti, furono acquisite da due nobili Filip Stein e Joseph Reiser che decisero di far fruttare i loro rispettivi appezzamenti, per lo più boschivi, frazionandoli "e vendendo i vari lotti con l'obiettivo di creare ex novo dei villaggi [...]" (Barzan, 2014, 57).

I due latifondisti proposero ufficialmente agli italiani, in particolare ai veneti e ai friulani, di acquistare i lotti del loro terreno, "consapevoli [...] della situazione di particolare povertà e di sovrappopolamento [...] che investiva quella zona [...] [,ma] fiduciosi della straordinaria attitudine al lavoro per cui gli abitanti di quelle zone erano celebri” (Barzan, 2014, 57).

La proposta fu accettata con entusiasmo da interi nuclei familiari, che si insediarono nei rispettivi lotti, incominciando a disboscare i terreni e, successivamente, con i mattoni prodotti, a realizzare le prime case e stalle:

Fra il marzo e l'aprile del 1880 le famiglie venete e friulane arrivarono in Slavonia. [...] [A]lcune furono destinate a popolare il podere di un latifondista e altre dovettero invece insediarsi in quello dell'altro. [...] Le famiglie bellunesi erano in totale quarantacinque e quarantatré si stabilirono nel podere di Raiser: le diciotto del Cadore in una zona mentre le venticinque provenienti da Casso, Castellavazzo, Longarone, Soverzene, Ponte nelle Alpi, in un'altra. In queste due zone i Bellunesi, cui si aggiunse qualche altra famiglia veneta e friulana, fondarono due distinti villaggi: i Cadorini Campo del Capitano, gli altri Kuen Novo Selo che poi venne chiamato Plostina. Con gli immigranti non avevano stipulato alcun contratto preventivo di compravendita dei terreni ma esistevano solo degli accordi sommari e delle promesse orali. In assenza di un preciso piano di insediamenti varato dalle autorità competenti si distribuirono quindi appezzamenti da disboscare in maniera arbitraria; [...]. In questa fase gli immigrati si costruirono baracche di legno e fango con tetti di paglia per ripararsi. Cominciarono ben presto a rivendicare la possibilità che era stata loro promessa di comprare il terreno e di gestirlo a loro piacimento e chiesero generi

[43] https://www.trevisotoday.it/cronaca/beggiato-emigranti-slavonia-novembre-2017.html. 
alimentari, medicinali, denaro per affrontare delle condizioni di miseria che stavano diventando insostenibili (Barzan, 2014, 61-62).

Le attività principali dei bellunesi erano l'agricoltura e l'allevamento, ma anche la produzione di carbone e di mattoni, tanto che nel XX secolo quest'ultima divenne prerogativa degli italiani di quella zona:

Con il passare degli anni gli Italiani cominciarono a incrementare la produzione di mattoni, aumentando il numero degli addetti e modernizzando le tecniche di lavorazione, impiegando anche appositi macchinari; l'attività diventò così, per tutto il ventesimo secolo, caratteristica degli Italiani della zona. Anche la produzione di carbone vegetale era già conosciuta dai Bellunesi emigrati, per cui fu riproposta anche in Slavonia (Barzan, 2014, 63).

Nel 1884 ci fu il riconoscimento ufficiale dei sette villaggi fondati dagli italiani in Slavonia ${ }^{[44]}$, pertanto gli immigrati divennero "titolari dei loro immobili e ricevettero delle agevolazioni [...] e inoltre sgravi e esoneri sul pagamento delle imposte"[45] (Barzan, 2014, 64).

Anche nell'area sudorientale della Serbia comprendente i distretti di Niš, Vranje, Pirot e Toplica si rileva a fine Ottocento, tra il 1884 e il 1888, una forte presenza "internazionale" di investitori ed appaltatori, ma anche di manodopera straniera impiegata nella costruzione "di 533 chilometri di strade ferrate", in particolare a Niš, in cui un po' tutti i paesi europei, tra cui l'Italia, aprirono i loro Consolati ${ }^{[46]}$.

Nel 1881, sempre in seguito alla crisi economica dell'Italia postunitaria, numerose famiglie italiane provenienti dal Friuli e dal Veneto decisero, pertanto, di emigrare anche in direzione della Serbia meridionale, soprattutto a Niš, in cui fervevano i lavori per la costruzione della rete ferroviaria e urgevano imprenditori e manodopera immigrata ${ }^{[47]}$.

[44] "[Q]uattro [insediamenti furono] fondati nella proprietà di Philippe Stain: Izidoravac, Trojeglava, Batinjani e un insediamento che era parte del paese di Lipik e tre che sorsero nel podere del latifondista Raiser" (Barzan, 2014, 63).

[45] "Il 15 giugno 1884 i rappresentanti dei sette villaggi, a nome dei loro compaesani, presentarono al Governo tramite il municipio di Pakrac la richiesta di cittadinanza austriaca" (Barzan, 2014, 64).

[46] Cfr. Genovesi-Bogicevic, Alessandra. Famiglie di origine italiana nell'area della Toplica. Disponibile da https://www.itals.it/famiglie-di-origine-italiana-nellarea-della-toplica.

[47] Ibidem. 


\section{L'emigrazione stagionale artigianale dal Bellunese verso i paesi dell'Europa centro-orientale: storia e memorie ${ }^{[48]}$}

Le fonti bibliografiche attestano che dalla metà dell'Ottocento si sarebbe verificata un'enorme richiesta di manodopera stagionale specializzata, proveniente dall'Italia nordorientale (soprattutto dal Bellunese e dal Friuli) e diretta principalmente verso i paesi dell'Europa centro e nordorientale.

In Austria, Ungheria, Boemia, Romania, Moldavia ${ }^{[49]}$, Bosnia, Bulgaria, ma anche in Prussia, Turchia, Russia, Siberia il personale sarebbe stato collocato nel ramo delle costruzioni edilizie, dello sviluppo ferroviario-stradale e nelle miniere, come muratore, scalpellino, tagliapietre o capomastro (cfr. Vendramini, 2001, 29, Wennemann, 1997, $41 ; 58 ; 75 ; 82$ e Scagno, 2008, 24-25; 27):

[...] nel 1893 [sarebbero stati] presenti [...] approssimativamente 1500 lavoratori italiani provenienti «in generale dalle provincie venete» [...] e [...] circa 1200 operai assunti come manovali, muratori, tagliapietre e fornaciai nei lavori pubblici civili e militari e nelle fabbriche di mattoni [...] e circa 220 operai impiegati nei lavori per la ferrovia [...] e il ponte di ferro sul Danubio [...]. Nel 1894, l'emigrazione stagionale superò i 5000 operai: [...]. Nel 1895, l'immigrazione temporanea nel distretto di Galati salì a 7000 individui [...], nella massima parte, del Veneto [...] [che solevano] venire in Rumania dalla fine di marzo a novembre inoltrato, cioè nell'epoca in cui la temperatura del paese permette il lavoro all'aria aperta, [...] (Scagno, 2008, 28-29).

Tra la metà e la fine dell'Ottocento, rinveniamo, a seguito degli uomini impiegati nella ristrutturazione delle vie di comunicazione stradali e ferroviarie, anche la presenza di donne e minori ${ }^{[50]}$ (cfr. Brunold, 1994, 251-253):

[48] Cfr. Campanale, 2006a, 42-90 e Campanale, 2019, 268-272.

[49] Nonostante l'immigrazione italiana in Moldavia fosse meno numerosa di quella di altre regioni del paese, si rilevano le medesime caratteristiche dell'immigrazione italiana nel resto della Romania, in riferimento alla provenienza degli emigrati (per lo più dal Veneto e dal Friuli), alla loro specializzazione (si trattava di muratori, minatori, imprenditori e appaltatori di edifici e opere pubbliche) e alla tipologia migratoria (molti di loro praticavano un'emigrazione stagionale e temporanea), nonché alla loro funzione di "mediatori culturali", capaci di fornire un significativo contributo allo sviluppo economico e culturale del paese in cui si erano trasferiti (cfr. Simion, 2018, 153-173).

[50] Cfr. Grandi, 2007, 22; 75; 166. 
Attorno a metà Ottocento alcune correnti di alpigiani cominciavano a raggiungere i cantieri delle grandi opere europee, aprendo una strada che li avrebbe portati ovunque si costruissero ferrovie; spesso li seguivano donne di famiglia che si prendevano cura di loro e di quanti potevano permettersi di pagare i servizi di lavanderia e cucina [...] (Grandi, 2007, 140).

Le testimonianze orali dei nostri intervistati ci confermano, che alla fine dell'Ottocento alcune donne, di solito le più giovani, sarebbero emigrate stagionalmente all'estero, a seguito degli uomini, per lavorare come cuoche per le imprese locali di costruzioni ${ }^{[51]}$ :

(1) Mia nonna paterna nel 1880, 1890 lavorava in Slesia come cuoca per le imprese che costruivano gallerie, ferrovie (U 57 STAG MONACO / DE MONACO - BL VODO - VI ARZIGNANO $)^{[52]}$

Si stima che, alla fine del XIX secolo, sarebbero emigrate dal Bellunese $^{[53]}$ 20.000/25.000 persone per lavorare temporaneamente e costantemente $^{[54]}$ oltralpe ${ }^{[55]}$, attratte dai maggiori guadagni (cfr. Culatti, 1997, 36-37 e Scagno, 2008, 51):

Non hanno preferenze per alcun determinato paese: accorrono là dove apprendono che esiste richiesta di mano d'opera al

[51] De Martini-Tihanyi $(1985,33-35 ; 41 ; 48)$ riconosce il ruolo fondamentale, svolto inizialmente dall'impresario Tallachini, nella costruzione della rete ferroviaria che si snodava attraverso tutto l'Impero: da Vienna, a Bratislava, Budapest, Brno, Praga. La studiosa fa riferimento anche all'emigrazione temporanea femminile di mogli, impiegate sia nei lavori di costruzione - di solito trasportavano con la gerla terra, sassi o sabbia - sia come lavandaie per le compagnie dei lavoratori.

[52] Gli esempi ricavati dall'analisi qualitativa (interviste "strutturate a risposta prefissata" e "semi-strutturate a risposta libera") del campione, estrapolato dalla mia tesi di dottorato precedentemente citata, verranno indicati tra parentesi tonde con un numero progressivo. Per la tutela della privacy si sono adottati i seguenti simboli per l'identificazione della provenienza e del tipo di migrazione dei soggetti. Tra parentesi si troverà indicato per primo il SESSO (U: uomo; D: donna), l'età in cifre, il tipo di migrazione (STAG: stagionale; PERM: permanente), la città tedesca, sede dell'attività, il luogo di nascita (ad esempio DE MONACO), la provenienza (BL VODO) e residenza (VI ARZIGNANO), con indicazione della provincia in sigle (ad esempio BL sta per Belluno). Nel caso in cui nascita, provenienza e residenza coincidano verrà data solo un'indicazione, ad esempio (U 55 STAG REUTLINGEN / BL VALLE) oppure (D 29 STAG MONACO / TV VITTORIO VENETO)

[53] Per un principio di "necessaria complementarità, i vuoti lasciati a valle dalla popolazione locale, emigrata stagionalmente oltralpe, in cerca di occupazioni più remunerative, erano colmati da un'«immigrazione contadina» (Brunold, 1994, 27), consentendo in tal modo la sopravvivenza e il difficile equilibrio della montagna veneta.

[54] "[I] lavoratori veneti emigra[va]no con l'intenzione di rimpatriare [e] rimpatria[va]no con l'intenzione di riemigrare" (Scagno, 2008, 51).

[55] Nel caso dell'Austria, la relativa vicinanza, nonché la semplicità delle documentazioni personali necessarie per l'espatrio avrebbero favorito l'espatrio (cfr. Culatti, 1997, 36-37 e Scagno, 2008, 51). 
salario migliore. Emigravano in Rumania prima del 1899; dopo, si diressero in Germania, in Isvizzera; ora ritornano in Rumania. Ho incontrato molti che avevano lavorato in Siberia, [...], alcuni [persino in] Cina. Ma di preferenza essi vanno in paesi non lontani dalla patria per poter ritornare ogni anno nei loro comuni di origine dove hanno la famiglia ed il campicello (Scagno, 2008, 51).

Tra le diverse tipologie migratorie dirette temporaneamente (di solito otto mesi) nei paesi dell'Europa centro-orientale, si segnalano gli "esamponari" (operai addetti alla costruzione delle linee ferroviarie), gli zattieri e gli "squarador", ossia "boscaioli con il compito di sagomare le piante" (Culatti, 1997, 39-41 e Scagno, 2008, 52-53) ${ }^{[56]}$.

Tali attività sono state espressamente menzionate anche da alcuni gelatieri stagionali veneti intervistati nell'ambito della mia tesi di dottorato precedentemente citata (vedi introduzione).

Sulla base delle suddette informazioni, nonché delle scarse fonti bibliografiche relative alla storia dell'emigrazione dei gelatieri veneti $^{[57]}$, il fenomeno può essere suddiviso, a grandi linee, in tre fasi. Per congruenza scientifica, noi delimiteremo la nostra analisi solo alla prima, che comprende il periodo che va dalla fine Ottocento fino agli inizi del secondo conflitto mondiale (1880-1915/1920-1939) e vede coinvolte le valli del Cadore e dello Zoldano.

Inizialmente, il flusso si diresse verso le città del Lombardo-Veneto, ma soprattutto verso Vienna e le altre capitali dell'Impero austro-ungarico, fino a coinvolgere presto anche i territori dell'Europa settentrionale, raggiungendo il Mar Baltico, l'ex Urss e la Prussia.

Tra le mete della prima ondata migratoria spiccano, secondo le memorie orali del nostro campione, soprattutto Vienna, ma anche Chemnitz, Budapest, Belgrado, Sarajevo, Riga, Breslavia, Katowice, Lipsia, Danzica.

In questo periodo, l'emigrazione dei gelatieri e la conseguente vendita del gelato sarebbero da collocare all'interno della "tradizione

[56] Verso la fine del secolo XIX, sono presenti in Austria, in Romania e in Caucaso zattieri bellunesi, mentre in Romania i cosiddetti "squarador" (cfr. Culatti, 1997, 39-41 e Scagno, 2008, 52-53).

[57] In merito alla storia dell'emigrazione dei gelatieri si veda Bortoluzzi, 1991, Caltran, 1999, Campanale, 2006a, Campanale, 2006b, Campanale, 2006c, Campanale, 2011, 31-58, così come Caldognetto e Campanale (a cura di), 2014, 231-244, Culatti, 1997, Mosena, 1996. 
secolare-di commerci ambulanti di vario genere-tipica dell'esodo stagionale montano ${ }^{[58]}$, passando dallo smercio di dolciumi, caldarroste, mandorle, frutti canditi alla vendita del gelato, distribuito inizialmente, nelle città italiane ed europee, con i famosi «carrettini»"(Campanale, 2019, 269-270):

(2) Inizialmente questa gente non vendeva gelato, ma venivano su per vendere croccanti e caldarroste, mandavano i ragazzi per le osterie a venderle, poi qualcuno ha iniziato col gelato (U 57 STAG MONACO / DE MONACO - BL VODO - VI ARZIGNANO)

Dall'analisi qualitativa, emerge, all'interno di suddetto flusso, la presenza di un'altra corrente migratoria. I veneti, diretti verso le città dell'Impero austro-ungarico, ma persino in Transilvania e nei Balcani, vennero impiegati per lo più nell'edilizia, come muratori, carpentieri, minatori o "squarador" (tagliaboschi):

(3) Mia nonna era nata a Sarajevo, il bisnonno verso la fine dell'Ottocento faceva lo squarador, faceva la stagione, andava via in estate e tornava in autunno. Mia mamma è nata in Austria perché il papà faceva l'intagliatore. Erano assunti da ditte locali per andare a lavorare nelle zone della Polonia, dell'exJugoslavia, della Prussia. Mio papà lavorava come falegname e d'estate come gelatiere a Bochum prima della seconda guerra. Con la guerra ha perso tutto, nel 1950 è tornato in Germania in Vestfalia a Dortmund [...] (U 53 STAG KEMPTEN / BL VALLE DI CADORE - VENAS)

Anche Scagno $(2008,52)$ rileva l'esistenza di tale categoria principalmente dopo il 1890, quando alcune "grandi società a capitale misto" reclutarono "mano d'opera proveniente in maggior parte dall'estero, dalla Transilvania e dalla Bucovina austriache e dall'Italia nord-orientale"

[58] Bovenkerk/Ruland (1992, 934 ss.) ritengono che il successo dei gelatieri fosse imputabile al fatto "di seguire la direzione dell'industrializzazione e di rispondere al potere d'acquisto della classe operaia. Gli artigiani offrivano prodotti che non erano richiesti sul mercato, anticipando prodotti nuovi e stimolando bisogni latenti; per questo motivo venivano ad occupare nella società d'accoglienza una posizione diversa nella scala sociale (spesso erano ai servizi delle classi più alte), costituendo quindi una piccola, ma compatta classe imprenditoriale. Il successo all'estero è stato ottenuto al di fuori di un processo d'assimilazione, così com'è stato nel caso dei gelatieri. La loro relativa indipendenza economica ha bloccato un eventuale processo di proletarizzazione, sebbene non si possa parlare di una vera e propria classe media" (Campanale, 2006a, 113). 
per "lo sfruttamento delle risorse forestali sui versanti orientale e meridionale dei Carpazi, e quindi all'interno del Regno di Romania":

Tagliaboschi e «segantini» erano ingaggiati direttamente nei paesi d'origine (Pontebba, Ponte Carnico, Ovaro, ma anche Longarone, Castellavazzo) dagli agenti delle Società per mezzo di un intermediario locale, e si recavano in vari gruppi in Romania in primavera ove rimanevano sino all'autunno inoltrato [...]. Abbiamo testimonianza documentaria sulla presenza stagionale pluriennale di consistenti gruppi di tagliaboschi friulani e bellunesi nei Carpazi meridionali: [...]; e nei Carpazi orientali in Moldavia [...] (Scagno, 2008, 52-53).

Soprattutto i tagliaboschi avrebbero goduto in Romania, negli ultimi anni prima dello scoppio della Grande Guerra, di "un meritato prestigio" per "abilità" tecnica, ma anche "per l'organizzazione del lavoro", nonché per "rapidità ed efficienza", tanto che "la loro attività [arricchì] in breve tempo la terminologia romena forestale di elementi veneti e retoromanzi” (Scagno, 2008, 53 e Campanale, 2019, 270).

Altri lavoratori stagionali bellunesi sarebbero stati, invece, reclutati, alla fine del XIX secolo, dai capimastri locali con svariate mansioni. Si contavano muratori, carpentieri, minatori, ad esempio per andare a lavorare nelle opere di costruzione della ferrovia transiberiana ${ }^{[59]}$, ma anche "eisenponer" ${ }^{60]}$ nelle zone d'influenza dell'Impero austro-ungarico, ossia in Transilvania ${ }^{[61]}$, Austria, Serbia, Croazia ${ }^{[62]}$, Ungheria, Romania, Slovacchia $^{[63]}$ (cfr. Vendramini, 2001, 29 e De Martini Tihany, 1985, 10-

[59] Secondo Vendramini $(2001,29)$ «l'inizio della vera emigrazione [edile di massa] dei contadini bellunesi per i lavori lontani» "si può far risalire alla prima metà dell'Ottocento grazie all'esportazione di manodopera del costruttore edile Tallachini, impegnato in opere pubbliche di rilievo come ad esempio la strada d'Alemagna o la costruzione della rete viaria e ferroviaria nel versante nord delle Alpi, specie in Austria, sulla linea ViennaTrieste. In quegli anni si venne a creare un meccanismo di reclutamento di manodopera a catena, a squadra e in parte specializzata, manodopera selezionata in loco, ossia nel Bellunese, secondo una rete di conoscenze e di esperienze precedentemente acquisite" (Campanale, 2006a, 49).

[60] Il termine "eisenponer" è la deformazione fonetica di "eisenbahner", vale a dire operai impiegati nella costruzione di ferrovie.

[61] La rete stradale della Transilvania ebbe inizio nel 1851 (cfr. http://emigrazionetrentina.museostorico.it/lagrande-emigrazione/).

[62] "Verso il 1860 iniziarono i lavori del tronco ferroviario Zigani Most-Zagabria, aperto al traffico fino a Sisak nell'ottobre 1862. Un'altra opera importante fu la linea Zagabria-Fiume" (Emigrazione italiana nei territori settentrionali dell'ex-Jugoslavia. Disponibile da http://brunodam.blog.kataweb.it/category/popolie-politiche/page/3/).

[63] Secondo De Martini-Tihanyi (1985, 10-11) “l'espansione del fenomeno verso i territori dell'Est potrebbe essere stata originata dallo spostamento di manodopera operaia, proveniente soprattutto dalle regioni alpine dell'Italia nordorientale, diretta, dalla seconda metà dell'Ottocento, verso i territori della Slovacchia e dell'Ungheria per trovare impiego come muratore e tagliapietre nella costruzione della rete ferroviaria e stradale". 
$\left.11^{[64]}\right)$ :

(4) I vecchi vendevano croccanti, caldarroste o frutti canditi con lo zucchero, come ambulanti d'inverno. Nel 1850 molti emigravano da Vodo di Cadore in Cecoslovacchia, in Polonia, a Lipsia, Danzica per lavorare nelle miniere o nelle industrie. Fino alla prima guerra mondiale stavano all'estero temporaneamente o stagionalmente. [...] (U 57 STAG MONACO / DE MONACO BL VODO - VI ARZIGNANO)

"Nello stesso periodo, ma in direzione opposta, ossia nella Germania dell'Ovest, troviamo [numerosi] bellunesi nelle province prussiane della Renania e Vestfalia, impiegati sia nell'industria mineraria, sia in opere edili o nella costruzione delle principali infrastrutture (canali, strade, vie ferrate)" (Campanale, 2006a, 49). I lavoratori, reclutati attraverso il sistema delle catene parentali, venivano organizzati in squadre costituite essenzialmente da reti paesane (cfr. Wennemann, 1997, 41; 58; 75; 82) ${ }^{[65]}$.

Sempre secondo il nostro campione, dalla fine dell'Ottocento fino alla prima guerra mondiale, gli uomini si sarebbero assentati da casa per tutto l'anno, facendo la cosiddetta “doppia stagione” (cfr. Mosena, 1996, 174; 250, Bortoluzzi, 1991, 231 e Pilcher, 1992, 17 $\left.{ }^{[66]}\right)$ :

(5) I vecchi lavoravano nelle miniere e sulle ferrovie. In Polonia a Chemnitz i miei vendevano gelato coi carrettini e nelle gelaterie. Facevano la doppia stagione. In estate vendevano gelato al Nord, mentre caldarroste al Sud in Germania, sempre come ambulanti. Se non avevano guadagnato abbastanza col gelato, restavano in Germania al Sud a vendere caldarroste [...] (D 48 STAG DINGOLFING / BL VALLE DI CADORE - VENAS)

(6) I vecchi vendevano croccanti, caldarroste o frutti canditi

[64] "L'abitudine degli alpigiani ad emigrare temporaneamente per contribuire alla realizzazione di opere edilizie prese forma durante il dominio napoleonico e sotto gli Austriaci si accentuò ulteriormente permettendo agli operai di specializzarsi via via nelle attività di: manovale, muratore, scalpellino, minatore. [...] Gli emigranti partivano all'inizio della primavera ma specialmente nel tardo inverno per poter coprire in tempo le distanze che li separavano dai luoghi dei lavori" (De Martini-Tihanyi, 1985, 32-33).

[65] Anche Wennemann $(1997,59)$ insiste sulla particolarità della migrazione italiana delle zone alpine, costituita essenzialmente da personale specializzato e da artigiani o piccoli commercianti, per cui l'attività stagionale all'estero rappresentava, non solo la fonte principale di guadagno, ma anche un determinato "modus vivendi" che si protraeva fino alla vecchiaia.

[66] La doppia veste migratoria è indicativa, secondo alcuni autori tra cui Pilcher $(1992,17)$, dell'abilità degli emigranti italiani in generale di riconoscere e di sfruttare le opportunità e l'evoluzione del mercato, specializzandosi nelle attività più redditizie, ma restando comunque all'interno di una "specializzazione etnica". 
con lo zucchero, come ambulanti d'inverno. Nel 1850 molti emigravano da Vodo di Cadore in Cecoslovacchia, in Polonia, a Lipsia, Danzica per lavorare nelle miniere o nelle industrie. Fino alla prima guerra mondiale stavano all'estero temporaneamente o stagionalmente (U 57 STAG MONACO / DE MONACO - BL VODO - VI ARZIGNANO)

Secondo Mosena $(1996,64)$ "con la fine della terza dominazione austriaca [...] [al]l'emigrazione estiva, prima di muratori, carpentieri, falegnami, boscaioli e successivamente di gelatieri ambulanti, [si sarebbe] affianca[ta] [...] quella invernale di fruttivendoli"[67].

Ciò viene confermato anche da Bortoluzzi (1989, 231), secondo cui nel 1871 "[i]l periodico La provincia di Belluno segnalava che in concomitanza del ritorno dei lavoratori dediti alle costruzioni ferroviarie, [...] prevalentemente durante il mese di novembre, si verificava la partenza di un'altra parte di popolazione occupata nel commercio ambulante e questi erano «i venditori di ciambelle, marroni e castagne dello Zoldano, i calderai e i vetrai del Cadore»:

[E]sistevano in queste valli persone con una duplice veste migratoria: durante la stagione estiva esercitavano il mestiere di muratore o scalpellino presso le imprese di costruzione edile nell'Impero asburgico; durante la stagione invernale, invece, lavoravano in proprio con il commercio ambulante di frutta cotta, cialde, e caldarroste nelle principali città padane. Questa duplice occupazione, che assicurava redditi complementari, per alcuni rimase un'abitudine fino a quando non si esaurirono i grandi lavori pubblici (ferrovie, strade) (Bortoluzzi, 1989, 231).

Una tale abitudine potrebbe essere stata motivata dal fatto che "con il passare degli anni e l'abitudine al trasferimento stagionale [oltralpe] [...] [sarebbe] cre[sciuta] la conoscenza delle varie opportunità d'impiego e dei settori più remunerativi [in cui] mettere a frutto le proprie abilità professionali e artigianali” (Scagno, 2008, 35).

[67] La tesi della doppia emigrazione è stata confermata dal "controllo incrociato dei passaporti per l'interno con quelli per l'estero, [...]. Gli stessi [zoldani] che dopo essere tornati dalla stagione invernale come fruttivendoli, emigravano in primavera verso le regioni dell'Impero, dichiaravano nel nuovo passaporto, una professione diversa. Genericamente venivano registrati come operai giornalieri, ma c'erano anche braccianti, falegnami, oppure fornai o chiodai. L'emigrazione non era esclusivamente estiva, c'era anche qualche venditore di frutta che si recava nelle città allora austriache" (Mosena, 1996, 214). 


\section{Conclusioni}

Con il presente contributo abbiamo voluto evidenziare la presenza di flussi migratori temporanei e stagionali provenienti dalle montagne del Bellunese e diretti verso i territori dell'Europa centro-orientale.

Dalla fine dell'Ottocento, all'emigrazione permanente transoceanica, si affiancò, nelle suddette zone montane, un altro movimento di massa stagionale o temporaneo, fortemente specializzato, di secolare tradizione e caratteristico di tutto l'arco alpino (Franzina, 1991, 49; 56).

Tra la fine dell'Ottocento e gli inizi del Novecento, numerosi nostri connazionali trovarono impiego oltralpe, soprattutto nel campo dell'edilizia e della ristrutturazione ferroviaria e stradale.

Nei paesi dell'Europa orientale i veneti lavorarono con svariate mansioni e in diversi ambiti (agricoltura, allevamento, attività boschive, miniere, costruzioni, infrastrutture). Furono apprezzati come manovali e artigiani specializzati, ma anche come impresari e abili architetti, tanto da insegnare alla popolazione locale il mestiere. Ricostruirono egregiamente città distrutte da eventi sismici, come Lubiana e Zagabria $\mathrm{o}$ ne rimodernizzarono altre, soprattutto in Romania, facendosi conoscere e stimare per la loro perseveranza, correttezza e abilità tecnica, a testimonianza [...] di una Regione [...] conosciut[a] all'estero, anche grazie ai suoi emigranti" (Campanale 2006c, 54 e Campanale, 2019, 273). 


\section{Bibliografia}

Bade, Klaus Jürgen (a cura di) (1992). Deutsche im Ausland - Fremde in Deutschland: Migration in Geschichte und Gegenwart. München: Beck Verlag.

Barzan, Guido (2014). Dialetto e cultura a Plostina, comunità bellunese in Croazia. Tesi di Laurea. Università degli Studi di Padova.

Bernardi, Ulderico, Todisco, Elisabetta (a cura di) (1998). Il dono dei migranti. Triveneti nel mondo. Cittadella (Padova): Biblos Edizioni.

Bortoluzzi, Tiziana (1991). Il flusso migratorio dei gelatieri bellunesi nell'area mitteleuropea. In Antonio Lazzarini \& Ferruccio Vendramini (a cura di), La montagna veneta in età contemporanea. Storia e ambiente. Uomini e risorse (229-244). Roma: Edizioni di Storia e Letteratura.

Bottin, Marco (2014). Cause economiche dell'emigrazione veneta nell'Ottocento. Tesi di Laurea. Università Ca' Foscari di Venezia.

Bovenkerk, Frank, Ruland, Loes (1992). Artisan Entrepreneurs: Two Centuries of Italian Immigration to the Netherlands. International Migration Revue, 26, 927-939.

Broch, Flavio (2010). Le parole dei kónže. Preliminari ad un'analisi etimologica del gergo dei seggiolai dell'Agordino. Tesi di Laurea. Università degli Studi di Verona.

Brunold, Ursus (a cura di) (1994). Gewerbliche Migration im Alpenraum. La migrazione artigianale nelle Alpi. Historikertagung in Davos. Convegno Storico di Davos, 25-27.IX.1991. Bozen: Verlagsanstalt Athesia.

Burmeister, Karl Heinz (1994). Einleitung. Introduzione. In Ursus Brunold (a cura di), Gewerbliche Migration im Alpenraum. La migrazione artigianale nelle Alpi. Historikertagung in Davos. Convegno Storico di Davos, 25-27. IX.1991 (7-14). Bozen: Verlagsanstalt Athesia.

Caldognetto, Maria Luisa, Campanale, Laura (a cura di) (2014). TRA INNOVAZIONE E TRADIZIONE - UN ITINERARIO POSSIBILE. Esperienze e proposte in ambito linguistico-letterario e storico-culturale per la didattica dell'italiano oltre frontiera. Atti delle Settimane della Lingua 
Italiana nel Mondo Università di Treviri, 2011 - 2012 - 2013 (123-140; 231244). Luxembourg: Edizioni Convivium.

Caltran, Tarcisio (a cura di) (1999). La storia del gelato: dall'epopea dei gelatieri alla Mostra Internazionale del Gelato. Caselle di Sommacampagna (VR): Cierre Grafica.

Campanale, Laura (2006a). I gelatieri veneti. Un'indagine sociolinguistica. Francoforte sul Meno: Peter Lang.

Campanale, Laura (2006b). L'emigrazione dei gelatieri italiani in Germania: la tradizione del gelato 'made in Italy'. In Thomas Krefeld (a cura di), Modellando lo spazio in prospettiva linguistica (157-172). Francoforte sul Meno: Peter Lang.

Campanale, Laura (2006c). I gelatieri veneti in Germania: un'indagine sociolinguistica. Altreitalie, 33, 45-64.

Campanale, Laura (2011). Emigrazione italiana in Germania $e$ bilinguismo. Evoluzione del fenomeno migratorio e implicazioni didattiche. Veitshöchheim: Wissenschaftlicher Verlag A. Lehmann.

Campanale, Laura (2019). Mobilità e migrazioni tra il Veneto e la Romania: un viaggio nella storia e nella memoria tra passato e presente. Quaestiones Romanicae, VII (2), 259-274.

Caritas Italiana (2008). Immigrazioni e lavoro in Italia. Statistiche, problemi e prospettive. Roma: IDOS.

Ceschi, Raffaello (1994). Migrazioni dalla montagna alla montagna. Migration von Berggebiet zu Berggebiet. In Ursus Brunold (a cura di), Gewerbliche Migration im Alpenraum. La migrazione artigianale nelle Alpi. Historikertagung in Davos. Convegno Storico di Davos, 25-27.IX.1991 (1582). Bozen: Verlagsanstalt Athesia.

Culatti, Davide (1997). Emigrazione e rientro. Il reinserimento lavorativo degli emigranti nel Bellunese. Rasai di Seren del Grappa (BL): Edizioni DBS.

De Martini-Tihanyi, Mirella (1985). L'emigrazione operaia dalle Venezie e dalla Lombardia alla Slovacchia. La costruzione delle ferrovie KosiceBohumin e Zvolen-Vrutky: 1870-1895. Padova: Erredici. 
Del Fabbro, René (1993). L’emigrazione proletaria italiana in Germania all'inizio del XX secolo. In Jens Petersen (a cura di), L'emigrazione tra Italia e Germania (27-115). Manduria: Pietro Lacaita Editore.

Grandi, Casimira (1994). Dalla Valsugana al Vorarlberg. Una storia di donne (1870-1915). Von der Valsugana nach Vorarlberg - Frauengeschichte (1870-1915). In Ursus Brunold (a cura di), Gewerbliche Migration im Alpenraum. La migrazione artigianale nelle Alpi. Historikertagung in Davos. Convegno Storico di Davos, 25-27.IX.1991 (293-392). Bozen: Verlagsanstalt Athesia.

Grandi, Casimira (2001). L'emigrazione femminile italiana in Germania: il perché di una scelta 1870-1914. Studi emigrazione, 38 (142), 346-374.

Grandi, Casimira (2007). Donne fuori posto. L'emigrazione femminile rurale dell'Italia postunitaria. Roma: Carocci Editore.

Franzina, Emilio (1991). L’emigrazione dalla montagna veneta fra Otto e Novecento. In Antonio Lazzarini \& Ferruccio Vendramini (a cura di), La montagna veneta in età contemporanea. Storia e ambiente. Uomini e risorse (185-228). Roma: Edizioni di Storia e Letteratura.

Franzina, Emilio (1991). Storia dell'emigrazione veneta. Verona: Cierre Edizioni.

Lazzarini, Antonio (1983). «Languire o fuggire»: alle origini dell'emigrazione veneta. In Emilio Franzina (a cura di), Un altro Veneto. Saggi e studi di storia dell'emigrazione nei secoli XIX e XX (19-32). Abano Terme (PD): Francisci Editore.

Mosena, Elisabetta (1996). La Val di Zoldo tra Otto e Novecento: popolazione, risorse ed emigrazione. Tesi di Laurea. Università Ca' Foscari di Venezia.

Palme, Rudolf (1994). Hauptströmungen der gewerblichen Migration in Nordtirol vom Spätmittelalter bis zur Jetztzeit. Principali correnti migratorie nel Nordtirolo dal tardo medioevo al presente. In Ursus Brunold (a cura di), Gewerbliche Migration im Alpenraum. La migrazione artigianale nelle Alpi. Historikertagung in Davos. Convegno Storico di Davos, 25-27.IX.1991 (225-260). Bozen: Verlagsanstalt Athesia. 
Pilcher, Edith (1991). «Pizza alla tedesca». Ein Literaturbericht zur Geschichte der italienischen Migration nach Deutschland. Ethnizität \& Migration, 2 (6), 5-25.

Pilcher, Edith (1992). Geschichte der italienischen Gewerbemigration nach Deutschland. Arbeitsheft, Berlin.

Pîrvu, Elena (2018). La migrazione italiana in Romania: aspetti socioculturali. In Carla Carotenuto, Edith Cognigni, Michela Meschini, Francesca Vitrone (a cura di), Pluriverso italiano: incroci linguisticoculturali e percorsi migratori in lingua italiana. Atti del Convegno internazionale Macerata-Recanati, 10-11 dicembre 2015 (635-646). Macerata: Eum edizioni Università di Macerata.

Rosoli, Gianfausto (a cura di) (1978). Un secolo di emigrazione italiana, 1876-1976. Roma: Centro studi emigrazione.

Scagno, Roberto (a cura di) (2008). Veneti in Romania. Ravenna: Longo Editore e Regione del Veneto.

Simion, Olivia (2018). Immigranti italiani nella Moldavia romena tra la fine dell'Ottocento e l'inizio del Novecento (1876-1916). Tesi di dottorato in Studi Storici. Università di Padova.

Trincia, Luciano (2001). Verso un quadro globale della diaspora italiana in Germania. Studi emigrazione, 38 (142), 245-258.

Vendramini, Ferruccio (a cura di) (2001). Sulle tracce del passato. Recuperi e documenti per una storia del Longaronese. Quaderno n. 2, Longarone (BL).

Wennemann, Adolf (1997). Arbeit im Norden: Italiener im Rheinland und Westfalen des späten 19. und frühen 20. Jahrhunderts. Osnabrück: Universitätsverlag Rasch. 


\section{Sitografia}

Baciu, Mirela. Quando i ,rumeni' erano gli italiani. Disponibile da http://www.blog2fete.com/quando-i-rumeni-erano-gli-italiani (consultato il 09 febbraio 2020).

Banfi, Emanuele. L'influsso dello spazio linguistico italiano sull'area balcanica. Disponibile da http://brunodam.blog.kataweb.it/category/ popoli-e-politiche/page/3/ (consultato il 18 febbraio 2020).

https://www.bellunesinelmondo.it/tag/plostine/ (consultato il 18 marzo 2020).

Bontempelli, Sergio. Quando gli italiani emigravano in Romania. Disponibile da http://www.sergiobontempelli.wordpress.com/2008/09/07/ italianiinromania/ (consultato il 09 febbraio 2020).

https://corrierealpi.gelocal.it/tempo-libero/2017/06/06/news/ viaggio-a-plostine-da-150-anni-enclave-bellunese-1.15455361) (consultato il 18 febbraio 2020).

De Matteo, Lynda. La Corsa verso la Romania degli imprenditori italiani. Disponibile da https://www.institutdelors.eu>2018/01 (consultato il 09 febbraio 2020).

Di Cesare, Valentina. La comunità italiana in Romania. Disponibile da http://www.lundici.it/2016/05/emigrazione-la-comunita-italiana-inromania/ (consultato il 09 febbraio 2020).

Emigrazione italiana nei territori settentrionali dell'ex-Jugoslavia. Disponibile da http://brunodam.blog.kataweb.it/category/popoli-epolitiche/page/3/ (consultato il 18 febbraio 2020).

http://emigrazionetrentina.museostorico.it/la-grande-emigrazione (consultato il 18 marzo 2020).

Genovesi-Bogicevic, Alessandra. Famiglie di origine italiana nell'area della Toplica. Disponibile da https://www.itals.it/famiglie-diorigine-italiana-nellarea-della-toplica (consultato il 18 febbraio 2020).

https://it.m.wikipedia.org/wiki/Slavonia (consultato il 18 marzo 2020).

Marzi, Alessio. Attraversati dai confini. Emigrare rimanendo (quasi) fermi. Disponibile da https://www.asei.eu/it/2017/03/attraversatidai-confini-emigrare-rimanendo-quasi-fermi/ (consultato il 18 febbraio 
2020).

Raluca Torre, Andreea. La migrazione italiana in Romania. Etnografia di un villaggio della Dobrugia. Disponibile da https://www. emigrazione-notizie.org/ (consultato il 09 febbraio 2020).

Rodica Chiretu, Marilena. Emigrazione italiana in Romania. Disponibile da https://www.pitestiromania.ro (consultato il 09 febbraio 2020).

https://www.trevisotoday.it/cronaca/beggiato-emigranti-slavonianovembre-2017.html (consultato il 18 marzo 2020).

\section{Summary}

The present contribution focuses on seasonal migrations from the Venetian mountains to the territories of the Middle-East Europe. In mid1800, a qualified group of seasonal migrants emigrated, especially from "Bellunese" to Eastern Europe; and were employed during the winter season for the construction of roads and railways or for woodcutting jobs. During the summer months, a large number of itinerant candy sellers joined this flow of emigration, to which the sale of ice cream subsequently took over. A trace of both of these flows of migration still remains in the documented oral accounts of Venetian ice-cream makers, to which they referred to as a unique and still active form of seasonal emigration, which have been practiced by the entire Northern-Veneto valley since 1880. First of all, they went to Vienna and to the neighboring territories of the Hapsburg Empire, then throughout Europe, especially in Germany immediately after the II $^{\circ}$ world war. This material was abstracted from my doctorate thesis entitled I gelatieri veneti in Germania. Un'indagine sociolinguistica (Venetian icecream makers in Germany: A sociolinguistic analysis) and published in 2006 by Peter Lang (Frankfurt). It is based on both qualitative and quantitative investigation. Furthermore, thanks to the authentic testimonies, it will be possible to take a "journey" down the memory lane of mountain valleys, that is dedicated to the seasonal craft emigration.

Key words: Veneto, Middle-East Europe, seasonal migration, historical professions, oral memory 
